SYMPOSIUM ON DRUGS

\title{
Nicotine conjugate vaccine: is there a right to a smoking future?
}

\author{
A Hasman, Søren Holm
}

J Med Ethics 2004;30:344-345. doi: 10.1136/jme.2002.001602

Tobacco consumption is believed to be one of the world's greatest preventable health problems. According to the World Health Organisation, 1.1 billion people worldwide are addicted to nicotine with tobacco causing an estimated four million premature deaths every year. The development of a nicotine conjugate vaccine suggests that immunisation may hold promise as a future therapeutic and preventive strategy for tobacco smoking and nicotine addiction. Allowing parents to immunise their children against smoking could be an infringement of children's right to an open future, however, and is not ethically unproblematic

$\mathrm{T}$ bacco consumption is believed to be one of the world's greatest preventable health problems. According to the World Health Organisation, 1.l billion people worldwide are addicted to nicotine with tobacco causing an estimated four million premature deaths every year. ${ }^{1}$ British biotech company Xenova Group is currently in a race with its American competitor, Nabi Biopharmaceuticals, to be the first to market a commercial vaccine against nicotine (Nabi Biopharmaceuticals press release: Nabi Biopharmaceuticals begins human testing of novel vaccine to fight nicotine addiction, 12 June 2002). The nicotine conjugate vaccine, known under the trade names NicVax and TA-NIC, has undergone phase I trials in humans, which show the drug is safe, well tolerated and generates a specific antinicotine immune response (Xenova group press release: Successful results of phase 1 trial for TA-NIC-first evaluation of antinicotine vaccine in man, 14 June 2002). These results suggest that immunisation may hold promise as a future therapeutic and preventive strategy for tobacco smoking and nicotine addiction. The nicotine conjugate vaccine acts by mobilising drug specific antibodies, which bind the nicotine molecules in the blood and prevent the drug from distributing to the brain, thereby reducing its behavioural effects. The nicotine addict will not experience any satisfaction from smoking and a first time user cannot become addicted.

Active immunisation is potentially irreversible (see below), but another possibility is passive immunisation by administration of an exogenously produced antibody. This gives temporary immunity but no permanent effect on the immune system. The antibody has a half life of over three weeks in humans, which makes it helpful to the smoker who wishes to quit the habit; lasting prevention, however, requires the vaccine in its active form . ${ }^{2}$ Work on animal models suggests that frequent booster injections or development of a depot formulation will be required for the active vaccine to create permanent effects. ${ }^{3}$ There are clear commercial and economic advantages to a vaccine which is irreversibly effective and such a drug could well result from further development. In the following we will assume that a vaccine formulation that creates permanent immunity to the effects of nicotine will be developed.

An active nicotine vaccine opens a range of new opportunities. First, the adult smoker who wants to quit the habit can ensure that cessation is effective (or at least not liable to interference by pleasurable effects of the occasional cigarette). This choice seems largely unproblematic from an ethical perspective. It can even be argued that the smoker has the right to have him- or herself vaccinated, even if immunisation is irreversible. Second, the vaccine offers an opportunity to prevent addiction among those who do not currently smoke. Research has shown that children and young adults are particularly susceptible to nicotine addiction $^{4}$ and could benefit if their parents have them vaccinated before they start experimenting with tobacco. A nicotine vaccine would be preventive in two ways: without the effect of nicotine the child would be less likely to experiment with smoking in the first place, and even if the child did decide to smoke, nicotine addiction would not follow, and s/he would find it easier to give up tobacco later in life. The vaccine would thus prevent drug dependence and a suggestion that children should be actively vaccinated against nicotine could seem to have some merit. ${ }^{5}$ This scenario requires a vaccine with long term effectiveness, since it is difficult in advance to predict exactly when a child is liable to start experimenting with smoking.

We have to ask, however, whether parents or others have a moral right to have children vaccinated against smoking. Although it is probably cheaper and more effective than offering to pay for driving lessons if they do not smoke, altering the immune system of children in order to modify future behaviour seems a major intrusion.

\section{A VACCINE LIKE ANY OTHER VACCINE}

Parents act in the best interests of the child and the rest of society on a number of occasions-some of which entail medical intervention. Thus, in most Western countries parents have the opportunity to have their children vaccinated against diphtheria, pertussis (whooping cough), measles etc. These are active vaccines, which prevent disease by irreversibly changing the immune system of the child, in a similar way to nicotine vaccines. As it is generally accepted that parents have a right (some people would even say a duty) to have their children immunised against disease, it could be argued that the same should by analogy be the case for nicotine vaccination. A number of considerations seem, however, to undermine this as a relevant analogy.

In vaccinating children against the diseases mentioned above we do not only benefit the individual child, we also create herd immunity, provided the level of vaccination is sufficiently high in the population. This means that the discomfort and risk of harm to the individual child is not only balanced by the direct benefits of having received the vaccination, but also by the extra protection offered by the inability of the infection to spread in the population. 
In the case of nicotine vaccination there is no direct herd immunity effect since tobacco use is not an infectious condition (and since the risk posed by passive smoking is probably minuscule). There may be an indirect social effect, if nicotine vaccination becomes popular and the number of young smokers decreases, but this would be a very peculiar social form of herd immunity.

Another argument against the analogy argues that use of nicotine is a lifestyle choice and not a disease; even if addiction to nicotine is considered a disease. Nicotine has both positive and negative consequences for health and social life, which the individual can assess and balance. People probably see nicotine's addictive effects as a negative outcome of smoking, but the drug's stimulating and appetite suppressing effects as positive outcomes. Some smokers find that the negative effects outweigh the positive effects but others do not. Whereas we might accept that an experienced nicotine user, who wants to stop smoking but cannot because of nicotine dependence, is suffering from a disease, it is more difficult to see how a child that has never been exposed to the drug, let alone given an opportunity to assess its benefits and disadvantages, can be in a similar position. Moreover, the beneficial effects of nicotine are not confined to its role as a stimulus; it has long been known that tobacco smoking offers protection against ulcerative colitis and gives a better prognosis for those afflicted by the disease. It is probably nicotine that causes this effect. ${ }^{6}$ Ulcerative colitis is an impairing disease, which is usually treated with high dose steroids, powerful immunosuppressants and surgery. There are severe side effects from this treatment and some patients feel that the potential overall benefits of smoking more than outweigh any adverse medical effects of the habit. Those who have been immunised against nicotine will not be able to get these benefits from the drug. Given the therapeutic uses of nicotine it is difficult to classify the misuse of the drug as a disease alongside other diseases such as diphtheria or whooping cough. Vaccinating a child against nicotine will not only prevent smoking but also restrict therapeutic options in later life.

The only aspect which seems to support an analogy between the nicotine vaccine and other vaccines is the fact that smoking causes disease. Causal links have been established between smoking and lung cancer, emphysema, and coronary disease; and smoking does lead to higher morbidity and mortality. If, however, we maintain that use of tobacco is a lifestyle choice rather than a disease we might point to a number of similar lifestyle choices which, although they are probably less addictive than smoking, also cause increased morbidity/mortality: alcohol consumption, excessive eating of fatty foods, and even mountaineering or other extreme sports are all lifestyle choices which increase morbidity or mortality one way or the other. Would we be willing to accept medical intervention in these lifestyle choices? It seems that the mere fact that the nicotine vaccine potentially prevents disease is insufficient justification for giving it to children.

Overall, nicotine immunisation appears very different from traditional vaccination. Active nicotine immunisation of children would raise very basic issues regarding the future opportunity of the individual. We will end by exploring these issues.

\section{MORAL RIGHT TO AN OPEN FUTURE}

In the ethical discussion of the limits of parental powers over children one argument has attained a central place. This argument was first proposed by the philosopher Joel Feinberg and claims that parental powers are limited by the child's right to have an open future. This entails that interventions that foreclose important possibilities in the future are prima facie wrong. Feinberg argues that while parents-for example, have a right to bring up their child in a particular religion or none, they do not have a right to have their child's education cut short on the basis that this will increase the chance that the child will not leave the religion in question. Having insufficient education significantly limits the child's future options and infringes its right to an open future. ${ }^{7} \mathrm{~A}$ right to an open future thus only relates to a relatively small fraction of parental choices. Parents are free to choose what languages their children learn and which school they attend (as long as they go to school) because parental discretion in such matters (a) does not impose restrictions on important options in later life (as those of us who have attained some degree of ability in a second language can attest to), and (b) does not affect choices that are truly open ended: a child has to learn some language, and what language it learns will depend just as much on its language community as on parental choice.

Does active nicotine vaccination infringe the right to an open future? As pointed out above active nicotine vaccination is irreversible and it does clearly limit the future options of the child. If vaccinated the child cannot become someone who smokes with the purpose of achieving the neurobehavioural effects of nicotine. Is this a significant reduction of future choices? Opinions are likely to differ on this question (and likely to mirror a priori held beliefs about whether smoking is a noxious habit or not), but it is important to note that smoking has a number of social functions, quite apart from the effects of the nicotine absorbed. For some it provides pauses and breathing spaces in a hectic daily life, for others it is an important part of group interactions. Active nicotine vaccination not only blocks the effects of nicotine, it also blocks the child from choosing to exploit the social functions of smoking.

It is therefore arguable that active nicotine vaccination is at the cusp of infringing the right to an open future, and that vaccination of children is not ethically unproblematic.

\section{Authors' affiliations \\ A Hasman, The Ethox Centre, University of Oxford, Oxford, UK \\ S Holm, Institute of Medicine, Law and Bioethics, University of Manchester, Manchester, UK \\ Conflicts of interest: None. Both authors are non-smokers.}

Correspondence to: Andreas Hasman, The Ethox Centre, University of Oxford, Old Road, Oxford, OX3 7L UK; andreas.hasman@green. oxford.ac.uk

\section{Accepted for publication 23 March 2003}

\section{REFERENCES}

1 World Heath Organisation. Fact sheet no 222. April 1999

2 Pentel PR, Malin DH, Ennifar S, et al A conjugate vaccine reduces nicotine distribution to brain and attenuates its behavioral and cardiovascular effects in rats. Pharmacol Biochem Behav 2000;65:191-8.

3 Hall W. The prospects for immunotherapy in smoking cessation. Lance 2002:360:1089-91.

4 Difranza JR, Savageau JA, Rigotti NA, et al. Development of symptoms of tobacco dependence in youth: 30 month follow up data from the DANDY study. Tob Control 2002; 11:228-35.

5 Cohen P. No more kicks: there are treatments on the way that promise to destroy the allure of addictive drugs for good. Problem solved? New Sci 2000;166:22-6.

6 Thomas GA, Rhodes J, Green JT, et al. Role of smoking in inflammatory bowel disease: implications for therapy. Postgrad Med J 2000;76:273-9.

7 Feinberg J. Freedom \& fulfilment-philosophical essays. Princeton, NJ:

Princeton University Press, 1992. 